\title{
Pancreatic Adenocarcinoma: Current Therapies and Challenges
}

\author{
Manpreet Sambi, ${ }^{1,2}$, Fiona Haxho ${ }^{1,2}$, Bessi Qorri' ${ }^{1}$, William Harless ${ }^{3 *}$ and Myron R Szewczuk ${ }^{1 *}$ \\ ${ }^{1}$ Department of Biomedical and Molecular Sciences, Queen's University, Canada \\ ${ }^{2}$ Contributing first authorship \\ ${ }^{3}$ ENCYT Technologies, Inc. Membertou, Canada
}

Submission: April 16, 2017; Published: April 19, 2017

"Corresponding author: Myron R Szewczuk, PhD, Department of Biomedical and Molecular Science, Queen's University, Kingston, ON K7L 3N6, Canada, Tel: +1-613-533-2457; Fax: +1-613-533-6796; Email: szewczuk@queensu.ca

\begin{abstract}
Gastrointestinal (GI) cancer is an all-encompassing term that refers to the forms of cancers of the digestive system including the esophagus, liver, gallbladder, stomach, small intestine, colon, rectum, anus and pancreas. Of the cancers mentioned, pancreatic ductal adenocarcinoma (PDAC) is the most deadly form of GI cancer owing partially to the late detection of this malignancy. At this point of diagnosis, the disease has metastasized and very few treatment options are available to patients. The aggressive nature of this cancer can be attributed to the substantial number of mutations acquired during its progression and its subsequent resistance to standard therapies such as chemotherapy and radiation. The heterogeneity of the subpopulations that are chemoresistant, particularly the tumor initiating population as known as cancer stem cells, make administrating conventional first-line treatments such as gemcitabine more difficult. Lastly, the tumor microenvironment and the early establishment of a metastatic niche by exosomes that facilitate dissemination of cancer cells to distant organs also contribute to the incurability of this type of cancer. Here we discuss the current clinical challenges in treating PDAC and the possible avenues that should be explored in order to improve current treatment options available to patients.
\end{abstract}

Keywords: Pancreatic cancer; PDAC; Cancer therapy; Drug resistance; Cancer stem cells; Alternative therapy

\section{Introduction}

Gastrointestinal (GI) cancers include the group of cancers that affect the digestive system such as the esophagus, gallbladder, liver, pancreas, stomach, small intestine, bowel colon and rectum, and anus. GI cancer is the most common form of cancer. In particular, pancreatic ductal adenocarcinoma (PDAC) accounts for the majority of pancreatic cancers and is one of the leading causes of cancer-related deaths worldwide [1]. With minimal improvements in treatment outcomes over the last forty years, lack of effective screening and early detection methods prevent clinicians from identifying PDAC in a premalignant stage [2], resulting in a five-year survival rate of only $5 \%$ following diagnosis $[3,4]$. Current treatment options include surgical resection, neoadjuvant and adjuvant chemotherapy and radiation. Unfortunately, heterogeneous cancer cell populations found in primary tumors and secondary micrometastases renders them resistant to cytotoxic therapies. To overcome these limitations, future therapies must target and disable the multiple enabling hallmarks that drive PDAC progression, immune-derived promoters of tumor development and growth, acquired drug resistance mechanisms, and pro-metastatic signals in the tumor microenvironment that potentiate cancer cell dissemination and homing to distant organs. Here, we provide a brief review of the current treatment options available for pancreatic cancer and ongoing challenges that need to be overcome to improve cancer therapy.

\section{Current Therapies and Limitations}

Although surgery remains one of the only curative treatment options, less than $20 \%$ of patients are surgical candidates [5]. Several poor predictors for successful resection are known: large tumor size [6], high tumor grade [7], positive tumor margins after surgical removal [5], elevated levels of CA 19-9 [8] and lymph node involvement [9]. Surgical PDAC patients remain at high risk for relapse and surgery has been shown to prolong survival by an average of only 10 months [10]. For patients with the advanced form of the disease that do not meet the criteria for surgery, radiation and chemotherapy are usually recommended as treatment options. Radiation therapy aims to eliminate rapidly 
proliferating cells in a specific area of the body by delivering a radioactive agent or high-energy rays that selectively induce DNA damage in dividing cells. Neoadjuvant radiation and chemotherapy therapy may be used to shrink an operable tumor prior to surgery. Adjuvant (post-surgery) radiation can also be used to treat the residual disease but the efficacy of this option is suboptimal due to its limited tolerance in normal tissue. The current standard of chemotherapy in the treatment of PDAC is gemcitabine (20,20-difluoro-20-deoxycytidine; dFdC), a nucleoside pyrimdine analog inhibiting DNA synthesis $[5,11]$. However, in non-surgical patients, gemcitabine treatment has been shown to prolong patient survival by only 4 months and is often used in palliation. Other neoadjuvant and adjuvant chemotherapeutics used today include flofirinox (5-fluorouracil, leucovorin, irinotecan, oxaliplatin) or a combination of gemcitabine with paclitaxel (abraxane), demonstrating tumor shrinkage in $20-30 \%$ of patients and slowing metastatic disease progression for approximately six months [12].

\section{Ongoing Clinical Challenges for Drug Development}

\section{Acquired Gene Mutations and Resistance to Therapy}

One of the greatest difficulties in preventing metastatic disease is reversing the acquired resistance to therapies. The prevalence of chemoresistance in PDAC has been linked to the acquisition of tumor-promoting genetic mutations, such as K-ras, p53, CDKN2a and SMAD4/DPC4 [13]. 90\% of PDAC patients also have KRAS2 point mutations, resulting in constitutively expressed Ras [14]. Once activated, Ras initiates a signaling cascade that activates cell proliferation and survival pathways, increasing cancer cell invasion [15]. Tumor-suppressor gene p53 is inactivated in approximately $80 \%$ of pancreatic tumors [16], resulting in impaired DNA damage recognition and repair, impaired apoptosis and deregulated mitosis [17]. Other tumorsuppressor genes encoded in the cdkn2a locus, including p16Ink4a and p15ARF, are present in about 90\% of human pancreatic cancers [18] and are implicated in drug-resistance mechanisms. The deregulated activities of specific membrane drug transporters also play a vital role in treatment efficacy. For example, human equilibrative nucleoside transporter-1 (hENT1) is a membrane facilitative transporter that is used by hydrophilic gemcitabine in order to enter cancer cells [19]. Without hENT1 activity, the rate of gemcitabine entry through the hydrophobic plasma membrane is negligible, contributing to gemcitabineresistance. As major drivers of drug resistance, these genetic and cellular factors present as targets for drug development and patient-specific predictors of treatment response.

\section{Clinical Implications of Pancreatic Intratumoral Heterogeneity}

Pancreatic tumor heterogeneity refers to the presence of multiple subpopulations postulated to be derived from a unique lineage of origin, within a single neoplasm [20]. The occurrence of a tumor-initiating population with the capacity to self-renew and give rise to differentiated progeny has become an area of intense investigation. In a comprehensive study assessing 24 different pancreatic cancers, results revealed an average of 63 genetic mutations per cancer, spanning 12 signal transduction pathways [21]. Intratumoral heterogeneity at the molecular level within pancreatic tumors has become of increasing interest because identifying unique biomarkers can be used as prognostic tools. K-ras mutations, for example, have been heavily investigated for their prognostic value because they represent an early stage in driver gene alterations that are required for disease progression [22]. However, the presence of this mutation in over $90 \%$ of pancreatic cancers, and studies demonstrating its detection in late-stage cancers, its value as a prognostic tool is diminished [23]. Despite its ineffective as a prognostic tool, when in addition to CDKN2A, TP53, BRCA2 and SMAD4/DPC4 mutations, they represent key genetic events that are required in order for PDAC to progress into an aggressive malignancy [22]. These key driver mutations are consistent across the majority of PDAC, erroneously providing a homogenous genetic profile; however, subpopulations within the tumor acquire their own unique genetic profiles as the architectural arrangement of subpopulations can also vary. For example, two different subpopulations can intermix, or they can be separated by a physical barrier (such as blood vessels) or by a difference in their microenvironment, both of which may generate differences in how these subpopulations respond to therapy [24]. Theoretically, because subpopulations within a tumor can be under the selective pressure of their microenvironment and barriers, each unique tumor cell population can have its own repertoire of potential therapeutic targets.

\section{Targeting Pancreatic Cancer Stem Cells}

Cancer stem cells (CSC) present another major obstacle in the treatment of PDAC. These dedifferentiated cells have been shown to maintain long-term tumorigenic potential, and are able to regrow new micrometastases [25], under the regulation of the surrounding tumor microenvironment (TME) [26]. Li et al. [25] identified a small population of pancreatic cancer stem cells with a characteristic CD44+/CD24+/ESA+ phenotype and constitute $0.2-0.8 \%$ of the pancreatic cancer stem cell population [25]. The mammalian target of rapamycin (mTOR) has been a potential target of interest due to its involvement in the proliferation of CSCs. Phosphorylation of the s6 ribosomal protein (s6rp) by p70s6 kinase, a downstream target of mTOR previously shown in the literature to be a reliable marker for the mTOR signaling pathway was found in only a small subset of pancreatic cancer cells $[27,28]$.

Rapamycin inhibition of mTOR resulted in a significant reduction of s6rp in pancreatic CSCs. However, the combination of cyclopamine (sonic hedgehog inhibitor), rapamycin, and gemcitabine (CRG) resulted in the elimination of virtually the entire CD133+ pancreatic CSC population [28]. This suggests that the combination of various therapeutic targets of CSC such as genes located in developmental pathways, such as hedgehog, 
Wnt, Notch, CXCR4 and Met, in combination with chemotherapy are capable of moderating the CSC pool. In addition, targeting apoptotic pathways such as DR5 and nodal-activin may also provide significant therapeutic benefit $[25,29]$. Nodal-activin are members of the TGF- $\kappa$ family, which are essential for embryonic stem cell (ESC) maintenance. Components of this signaling cascade are over expressed in pancreatic CSCs, with nodal highly expressed in pancreatic cancer tissue during the development and progression of PDAC, but not detectable in normal pancreatic tissue. Nodal inhibition was able to chemosensitize inherently chemoresistant pancreatic CSCs to therapy [30]. These findings support the notion that pancreatic intratumoral heterogeneity and CSCs are major enabling components that contribute to drug resistance and disease progression. A better understanding of CSC populations and how they interact with one another and the surrounding TME will enable further progress in the treatment of pancreatic cancer.

\section{Exosomes in Establishment of the Metastatic Niche}

PDAC is characterized by its late detection, which may be correlated with its high metastatic potential. Current screening methods lack the sensitivity to detect early onset with $80 \%$ of PDAC patients presenting with metastases at the time of diagnosis [31]. Metastatic disease is difficult to treat with conventional chemotherapeutic methods due to their unique genetic repertoire, size and location within a tissue relative to the primary tumor [32]. Therefore, it is important to elucidate the molecular events that mediate the development of metastatic disease. Exosomes are extracellular membrane-bound vesicles containing nucleic acids and proteins [31]. Exosomes have been implicated in the establishment of a premetastatic niche in liver metastases of patients with PDAC [33] and have been proposed as early detection tools as they circulate in the bloodstream, which make them an important area of study [31].

Exosome-mediated development of a metastatic TME is proposed to involve the release of PDAC exosomes containing macrophage migration inhibitor factor (MIF) to preferentially fuse with Kupffer Cells (KC) of the liver [33]. This leads to KC secreting TGF- $\beta$, which promotes the release of fibronectin by hepatic stellate cells, generating a pro-inflammatory microenvironment and recruiting macrophages [33]. Inhibition of MIF leads to the elimination of the liver premetastatic niche and prevents metastasis [31]. Recently glypican-1 (GPC1), a cancer exosome surface proteoglycan, has been investigated as an early detection biomarker as it is enriched on the surface of exosomes released from primary tumors. Its expression is also correlated with the presence of K-ras mutations found in pancreatic lesions that are undetectable by conventional means [31]. The efficacy of exosomes as mediators of metastasis has important implications on early detection strategies and possible therapeutic targets as they circulate the blood and are known mediators of intercellular communication.
Paracrine signals from pancreatic tumor cells are able to induce a desmoplastic reaction, characterized by the extracellular proliferation of leukocytes, fibroblasts, endothelial cells, neuronal cells, and production of collagen type I and hyaluron [34]. Desmoplasia results in the formation of a thick stromal environment around pancreatic cancer cells [34] resulting in a mechanical barrier for drug delivery and is thought to contribute to the anti-angiogenic and hypoxic environment characteristic of PDAC, supporting tumor formation, progression and metastasis [35]. Studies have demonstrated that desmoplasia-promoting signals originate from the K-ras mutant oncogene in the tumor epithelium [36]. Sonic hedgehog (SHH) is also overexpressed in pancreatic cancer and results in the induction of extracellular fibroblasts, contributing to their growth and differentiation [36]. Furthermore, growth factors such as TGF- $\beta$, platelet-derived growth factor (PDGF) and fibroblast growth factor (FGF) activate pancreatic stellate cells into myofibroblasts capable of secreting ECM components that further reduce the vascularization of the primary tumor [37]. Many proteins expressed by stromal cells have been correlated with poor prognosis and drug resistance, including COX-2, PDGF receptor, vascular endothelial growth factor (VEGF), stromal-derived factor (SDF), chemokines, integrins, secreted protein acidic and rich in cysteine (SPARC), and SHH elements [35,37].

\section{Immune-Regulated Tumorigenesis}

Notably, the dense stroma is characterized by a tumorpromoting immunosuppressive environment. Using a CD40 antibody combined with gemcitabine therapy, researchers have attempted to reverse immune suppression and drive anti-tumor T-cell responses in patients with non-resectable pancreatic cancer. Studies have shown that this dual combination results in tumor regression by stimulating tumor-associated macrophages (TAM) to attack and degrade the stroma [38]. To date, PDAC treatment has proved most effective in patients with locally advanced disease, particularly in patients with wild-type DPC4 tumors, as they are known to be less prone to metastasis and possess higher stromal content. However, primary tumors that have already metastasized cannot be effectively treated with stromal-targeting agents because distant metastases arising from this cancer do not have a hypovascularized stroma [39]. Several cell types involved in the desmoplastic reaction, including TAMs, cancer associated fibroblasts, regulatory T-cells and myeloid derived suppressor cells contribute to tumorigenesis. K-ras-dependent signaling molecules have also been shown to up regulate granulocyte-macrophage colony stimulating factor (GM-CSF), thus promoting the maturation of myeloid progenitor cells into myeloid derived suppressor cells [40].

Unlike the stroma, the primary pancreatic tumor and/or distant micrometastases can be exposed to a highly inflammatory microenvironment. Cancer-associated inflammation can contribute to drug resistance, selection of CSCs and desmoplasia. Nuclear factor kappa B (NFkB) signaling, critical for the 
inducible expression of cellular and viral genes involved in inflammation, has been found to be constitutively activated in pancreatic cancer cells [41]. Inducible cyclooxygenase (COX)-2, a downstream target gene of NF- $\mathrm{BB}$, is not normally expressed but can be up regulated by cytokines, growth factors and certain tumor-promoter genes. It is involved in prostaglandin synthesis, promotion of angiogenesis, immune evasion and inhibition of apoptosis. COX-2 has been found to be overexpressed in pancreatic adenocarcinomas and is localized to the cytoplasm of the tumor cells as opposed to the surrounding stromal or immune cells [41].

\section{Conclusion}

Several clinical challenges remain in the treatment of pancreatic cancer. In addition to its advanced-stage detection, there have been no significant improvements in patient survival rates or in the development of effective therapies. These challenges arise from the understanding that the cancer cell program is adaptive, self-sustaining and invasive, and that despite therapy, aggressive phenotypes will survive and metastasize. Thus, future studies must not rely on targeting a single oncogenic or mitotic pathway, but must suppress the multiple stages of tumorigenesis of pancreatic cancer cells and their enabling microenvironment.

\section{Acknowledgments}

This work was supported in part by grants to MR Szewczuk from the Natural Sciences and Engineering Research Council of Canada, private sector cancer funding from the Josefowitz Family to MR Szewczuk and Encyt Technologies, Inc.

M. Sambi is a recipient of the Queen's Graduate Award (QGA). F. Haxho was the recipient of the QGA, the Graduate Entrance Tuition Award (GETA), the Natural Sciences and Engineering Research Council of Canada (NSERC) Alexander Graham Bell Canada Graduate Scholarship-Master's (CGS M) and now the Vanier Canada Graduate Scholarship. The authors report no conflicts of interest in this work.

\section{References}

1. Jemal A, Bray F, Center MM, Ferlay J, Ward E, et al. (2011) Global cancer statistics. CA Cancer J Clin61(2): 69-90.

2. Sener SF, Fremgen A, Menck HR, Winchester DP (1999) Pancreatic cancer: a report of treatment and survival trends for 100,313 patients diagnosed from 1985-1995, using the National Cancer Database. J Am Coll Surg 189(1): 1-7.

3. Stathis A, Moore MJ (2010) Advanced pancreatic carcinoma: current treatment and future challenges. Nat Rev Clin Oncol 7(3): 163-172.

4. Matsuda T, A Matsuda (2014) Five-year relative survival rate of pancreas cancer in the USA, Europe and Japan. Jpn J Clin Oncol 44(4): 398-399.

5. Rossi ML, Rehman AA, Gondi CS (2014) Therapeutic options for the management of pancreatic cancer. World J Gastroenterol 20(32) 11142-1159.

6. Fortner JG, Klimstra DS, Senie RT, Maclean BJ (1996) Tumor size is the primary prognosticator for pancreatic cancer after regional pancreatectomy. Ann Surg 223(2): 147-153.
7. Wasif N, Ko CY, Farrell J, Wainberg Z, Hines OJ, et al. (2010) Impact of tumor grade on prognosis in pancreatic cancer: should we include grade in AJCC staging? Ann Surg Oncol 17(9): 2312-2320.

8. Goonetilleke KS, Siriwardena AK (2007)Systematic review of carbohydrate antigen (CA 19-9) as a biochemical marker in the diagnosis of pancreatic cancer. Eur J Surg Oncol 33(3): 266-270.

9. Riediger H, Keck T, Wellner U, zur Hausen A, Adam U, et al. (2009) The lymph node ratio is the strongest prognostic factor after resection of pancreatic cancer. J Gastrointest Surg 13(7): 1337-1344.

10. Bilimoria KY, Bentrem DJ, Ko CY, Ritchey J, Stewart AK, et al. (2007) Validation of the 6th edition AJCC Pancreatic Cancer Staging System: report from the National Cancer Database. Cancer 110(4): 738-744.

11. Huang MT, Lysz T, Ferraro T, Abidi TF, Laskin JD, et al. (1991) Inhibitory effects of curcumin on in vitro lipoxygenase and cyclooxygenase activities in mouse epidermis. Cancer Res 51(3): 813-819.

12. Marsh Rde W, Talamonti MS, Katz MH, Herman J (2015) Pancreatic cancer and FOLFIRINOX: a new era and new questions. Cancer Med 4(6): 853-863.

13. Almoguera C, Shibata D, Forrester K, Martin J, Arnheim N, et al. (1988) Most human carcinomas of the exocrine pancreas contain mutant $\mathrm{c}-\mathrm{K}-$ ras genes. Cell 53(4): 549-554.

14. Hruban RH, Maitra A, Goggins M (2008) Update on pancreatic intraepithelial neoplasia. Int J Clin Exp Pathol 1(4): 306-316.

15. Gedde-Dahl T, Eriksen JA, Thorsby E, Gaudernack G (1992) T-cell responses against products of oncogenes: generation and characterization of human T-cell clones specific for p21 ras-derived synthetic peptides. Hum Immunol 33(4): 266-274.

16. Olive KP, Tuveson DA, Ruhe ZC, Yin B, Willis NA, et al. (2004) Mutant p53 gain of function in two mouse models of Li-Fraumeni syndrome. Cell 119(6): 847-860.

17. Caldas C, Hahn SA, da Costa LT, Redston MS, Schutte M, et al. (1994) Frequent somatic mutations and homozygous deletions of the p16 (MTS1) gene in pancreatic adenocarcinoma. Nat Genet 8(1): 27-32.

18. Hahn SA, Hoque AT, Moskaluk CA, da Costa LT, Schutte M, et al. (1996) Homozygous deletion map at 18q21.1 in pancreatic cancer. Cancer Res 56(3): 490-494.

19. Nordh S, Ansari D, Andersson R (2014) Andersson, hENT1 expression is predictive of gemcitabine outcome in pancreatic cancer: a systematic review. World J Gastroenterol 20(26): 8482-8490.

20. Heppner GH (1984) Tumor heterogeneity. Cancer Res 44(6): 22592265.

21. Jones S, Zhang X, Parsons DW, Lin JC, Leary RJ, et al. (2008) Core signaling pathways in human pancreatic cancers revealed by global genomic analyses. Science 321(5897): 1801-1806.

22. Bardeesy N, DePinho RA (2002) DePinho, Pancreatic cancer biology and genetics. Nat Rev Cancer 2(12): 897-909.

23. Le Calvez-Kelm F, Foll M, Wozniak MB, Delhomme TM, Durand G, et al. (2016) KRAS mutations in blood circulating cell-free DNA: a pancreatic cancer case-control. Oncotarget 7(48): 78827-78840.

24. Burrell RA (2013) The causes and consequences of genetic heterogeneity in cancer evolution. Nature 501(7467): 338-345.

25. Li C, Heidt DG, Dalerba P, Burant CF, Zhang Let al. (2007) Identification of pancreatic cancer stem cells. Cancer Res 67(3): 1030-1037.

26. Dean M, Fojo T, Bates S (2005) Tumour stem cells and drug resistance. Nature Reviews Cancer 5(4): 275-284.

27. Aoki M, Blazek E, Vogt PKA (2001) role of the kinase mTOR in cellular transformation induced by the oncoproteins P3k and Akt. Proceedings of the National Academy of Sciences 98(1): 136-141. 
28. Mueller MT, Hermann PC, Witthauer J, Rubio-Viqueira B, Leicht SF, et al. (2009) Combined Targeted Treatment to Eliminate Tumorigenic Cancer Stem Cells in Human Pancreatic Cancer. Gastroenterology 137(3): 1102-1113.

29. Rhim AD, Mirek ET, Aiello NM, Maitra A, Bailey JM, et al. (2012) EMT and dissemination precede pancreatic tumor formation. Cell 148(1-2): 349-361.

30. Lonardo E, Hermann PC, Mueller MT, Huber S, Balic A, et al. (2011) Nodal/Activin Signaling Drives Self-Renewal and Tumorigenicity of Pancreatic Cancer Stem Cells and Provides a Target for Combined Drug Therapy. Cell Stem Cell 9(5): 433-446.

31. Melo SA, Luecke LB, Kahlert C, Fernandez AF, Gammon ST, et al. (2015) Glypican-1 identifies cancer exosomes and detects early pancreatic cancer. Nature 523(7559): 177-182.

32. Schroeder A, Heller DA, Winslow MM, Dahlman JE, Pratt GW, et al. (2011) Treating metastatic cancer with nanotechnology. Nat Rev Cancer 12(1): 39-50.

33. Costa-Silva B, Aiello NM, Ocean AJ, Singh S, Zhang H, et al. (2015) Pancreatic cancer exosomes initiate pre-metastatic niche formation in the liver. Nat Cell Biol 17(6): 816-826.

34. Neesse A, Algül H, Tuveson DA, Gress TM (2015) Stromal biology and therapy in pancreatic cancer: a changing paradigm. Gut 64(9): 14761484.

35. Zhang GN, Liang Y, Zhou LJ, Chen SP, Chen G, et al. (2011) Combination of salinomycin and gemcitabine eliminates pancreatic cancer cells.
Cancer Lett 313(2): 137-144.

36. Tian H, Callahan CA, DuPree KJ, Darbonne WC, Ahn CP, et al. (2099) Hedgehog signaling is restricted to the stromal compartment during pancreatic carcinogenesis. Proc Natl Acad Sci USA 106(11): 4254-4259.

37. Jimeno A, Feldmann G, Suárez-Gauthier A, Rasheed Z, Solomon A, et al. (2009) A direct pancreatic cancer xenograft model as a platform for cancer stem cell therapeutic development. Mol Cancer Ther 8(2): 310314.

38. Beatty GL, Chiorean EG, Fishman MP, Saboury B, Teitelbaum UR, et al. (2011) CD40 agonists alter tumor stroma and show efficacy against pancreatic carcinoma in mice and humans. Science 331(6024): 16121616.

39. Schrader H, Wiese M, Ellrichmann M, Belyaev O, Uhl W, et al. (2012) Diagnostic value of quantitative EUS elastography for malignant pancreatic tumors: relationship with pancreatic fibrosis. Ultraschall Med 33(7): E196-E201.

40. Bayne LJ, Beatty GL, Jhala N, Clark CE, Rhim AD, et al. (2001) Tumorderived granulocyte-macrophage colony-stimulating factor regulates myeloid inflammation and $\mathrm{T}$ cell immunity in pancreatic cancer. Cancer Cell 21(6): 822-35.

41. Tucker ON, Dannenberg AJ, Yang EK, Zhang F, Teng L, et al. (1999) Cyclooxygenase-2 Expression Is Up-Regulated in Human Pancreatic Cancer. Cancer Research 59(5): 987.

\section{Your next submission with JuniperPublishers will reach you the below assets}

- Quality Editorial service

- Swift Peer Review

- Reprints availability

- E-prints Service

- Manuscript Podcast for convenient understanding

- Global attainment for your research

- Manuscript accessibility in different formats

( Pdf, E-pub, Full Text, audio)

- Unceasing customer service

Track the below URL for one-step submission https://juniperpublishers.com/online-submission.php 